\title{
La collection Michel Bernstein (Université Senshu, Tokyo)
}

Yoshiaki Ômi et Michel Biard

\section{Q OpenEdition \\ 1 Journals}

Édition électronique

URL : https://journals.openedition.org/ahrf/12047

DOI : 10.4000/ahrf.12047

ISSN : 1952-403X

Éditeur :

Armand Colin, Société des études robespierristes

Édition imprimée

Date de publication : 1 juin 2011

Pagination : 193-196

ISSN : 0003-4436

\section{Référence électronique}

Yoshiaki Ômi et Michel Biard, « La collection Michel Bernstein (Université Senshu, Tokyo) », Annales

historiques de la Révolution française [En ligne], 364 | avril-juin 2011, mis en ligne le 01 juin 2014, consulté le 23 avril 2022. URL : http://journals.openedition.org/ahrf/12047 ; DOI : https://doi.org/ 10.4000/ahrf.12047 


\title{
LA COLLECTION MICHEL BERNSTEIN (UNIVERSITÉ SENSHU, TOKYO)
}

\author{
Yoshiaki ÔMI et Michel BIARD
}

En 1977, à l'occasion du centenaire de sa fondation, l'Université Senshu, l'une des principales universités de Tokyo, a fait l'acquisition de la collection rassemblée au fil de plusieurs décennies par le Français Michel Bernstein ${ }^{1}$. Celui-ci, en 1979, a résumé dans une lettre (publiée ci-après), adressée à l'Université Senshu, tout à la fois les raisons de son intérêt pour la Révolution française et les diverses étapes qui l'avaient conduit à créer sa collection. Cette lettre ${ }^{2}$, quoique brève, est un merveilleux témoignage de la transmission d'une culture révolutionnaire fondée, entre autres, sur la référence à la "Grande Révolution », mais elle permet aussi de comprendre comment il était encore possible dans les années 1920-1930 d'acheter à Paris, et ailleurs, de nombreuses brochures et des journaux publiés au cours de la Révolution française.

La Collection Michel Bernstein est aujourd'hui conservée dans la réserve des ouvrages précieux de la bibliothèque de l'Université Senshu,

(1) Né le 13 janvier 1906 à Lyon, décédé le 15 août 2003 à Villefargeau (Yonne), Michel Bernstein a notamment été le cofondateur avec Léon Centner (1919-2002), en 1966, des éditions Edhis qui publièrent de nombreux reprints, dont certains fondés sur sa propre collection révolutionnaire. Sur ce personnage, outre la lettre publiée ci-après, voir la notice rédigée par Michel Cordillot dans la version électronique du Maitron (Dictionnaire biographique du mouvement ouvrier français [...]. Michel Cordillot est également l'auteur de la notice nécrologique publiée dans le journal Libération le $1^{\text {er }}$ septembre 2003). Ce texte ne figure pas dans l'édition papier du Maitron, mais se trouve dans le CD-ROM qui accompagne le volume de la nouvelle série comprenant la lettre B.

(2) Cette lettre a fait l'objet d'une première publication, en français et en japonais, dans une revue éditée par l'Université Senshu : Bernstein-Bunko Dayori (Bulletin de la Collection Michel Bernstein), no 1, 1980, p. 5-11. 
sur le campus d'Ikuta (l'un des deux campus de l'Université), dans la proche banlieue sud-ouest de Tokyo. Pendant une trentaine d'années, un long et minutieux travail de classement et de catalogage a été entrepris, cependant que des microfilms étaient réalisés. Aujourd'hui, le catalogue imprimé de la Collection (en neuf volumes), dont un exemplaire a été déposé à la Bibliothèque nationale de France, permet aux chercheurs une première découverte des fonds disponibles. Désormais, l'Université Senshu entre dans une autre phase de son projet et entend notamment établir à terme un catalogue général, avec des index informatisés qui permettront aux chercheurs du monde entier de suivre les pistes offertes par ce fonds. Elle a également entrepris une importante série de publications, tels les volumes des Opinions des conventionnels lors du procès de Louis XVI ${ }^{3}$.

Le fonds Michel Bernstein se compose de près de 40000 brochures publiées à l'époque révolutionnaire, consulaire et impériale, mais aussi dans les années précédant 1789 (beaucoup de documents concernent les Parlements); d'une très importante collection de journaux et feuilles volantes d'époque révolutionnaire (de très belles séries complètes - dont le Moniteur dans sa version d'origine -, ainsi que des numéros isolés); des procès-verbaux imprimés des Assemblées successives et des Collections imprimées des lois; d'environ 2000 pièces manuscrites (auxquel les s'ajoutent de nombreux imprimés sur lesquels sont portées des mentions manuscrites); enfin d'un grand nombre de livres, outils de recherche, thèses, tirés-à-part d'articles, etc., concernant la Révolution française. Comme l'expliquait Michel Bernstein dans sa lettre de 1979, il s'agit là assurément d'une des principales collections privées portant sur la Révolution française. Dès lors, l'Université Senshu peut aujourd'hui fort légitimement s'enorgueillir de posséder l'un des fonds révolutionnaires parmi les plus prestigieux au monde.

Plusieurs sondages ont été opérés afin de repérer quelle part de ce fonds semblait, sinon composée de pièces uniques, à tout le moins de documents non conservés à la Bibliothèque nationale et dans les grandes

(3) Opinions des Conventionnels sur le Jugement de Louis XVI. Recueil des Documents sur la Révolution française conservés à la Bibliothèque de Michel Bernstein de l'Université SENSHU, Première Série, 6 vol., 2008. 
bibliothèques municipales de France ${ }^{4}$. S'il est impossible de donner à ce stade de l'enquête une estimation raisonnable de cette part, force est de constater la richesse exceptionnelle de la Collection Michel Bernstein et son immense intérêt pour la communauté scientifique. Le professeur Yoshiaki Ômi, en charge de cette Collection à l'Université Senshu, a d'ores et déjà réalisé plusieurs travaux sur des pièces inédites. Il a notamment publié le cahier du tiers état de la ville du Puy, dont un exemplaire manuscrit figure dans la Collection ${ }^{5}$. À son initiative, ont également été entreprises diverses publications de sources numérisées, dont la série des Opinions sur le procès de Louis XVI mentionnée ci-dessus. D'autres projets sont naturellement aujourd'hui à l'étude.

Les Annales historiques de la Révolution française se devaient de faire connaître à tous l'existence de cette exceptionnelle Collection Michel Bernstein. Nous avons choisi de publier pour l'occasion la lettre, en tout point passionnante, de ce dernier, ainsi que quelques exemples de documents manuscrits figurant dans un dossier consacré à Félix Le Peletier (deux documents sur l'ordre d'arrestation porté contre lui au moment de la répression contre les Babouvistes en l'an IV, ainsi qu'une

(4) La chercheuse catalane Maria Betlem Castellà Pujols a été invitée à deux reprises ces dernières années par l'Université Senshu pour effectuer un travail de comparaison du fonds Bernstein avec les fonds français grâce aux ressources de l'Internet. Hélas, elle ne s'est guère fait écho en France de ce travail, alors qu'il est pourtant évidemment fondamental de faire découvrir l'existence de la très riche Collection Bernstein à l'ensemble de ceux qui, de par le monde, effectuent des recherches sur la Révolution française. Ses conclusions attestent qu'un tiers des documents sur lesquels portait son sondage (1000, puis 4000 autres, documents) ne sont pas conservés à la BNF et dans les grandes bibliothèques françaises. Pour autant, cela n'en fait naturellement pas des pièces devenues rarissimes, puisque un bon nombre d'entre eux sont sans doute présents dans d'autres bibliothèques (y compris hors de France) et surtout dans les fonds d'Archives (AN, AD, AM). Pour autant, la probabilité n'en reste pas moins importante que beaucoup des documents aujourd'hui conservés par l'Université Senshu soient des pièces très rares. Enfin, même si des manuscrits d'époque révolutionnaire existent souvent en plusieurs exemplaires dès leur apparition (un arrêté - ou une lettre - d'un représentant du peuple en mission est le plus souvent copié par tous ceux qui en sont informés et a fortiori par les citoyens chargés d'exécuter les décisions), il va de soi que la majorité des documents manuscrits de la Collection est constituée de pièces uniques. Maria Betlem Castellà Pujols, « La valeur et le caractère historique de la Collection des documents de Michel Bernstein : une analyse du tome 6 du catalogue ", dans The Michel Bernstein Collection and Studies on the French Revolution, Center for Historical Studies, Institute for Development of Social Intelligence, Senshu University, 2008, p. 19-37; id., " Au-delà de la rareté... des merveilles. Deuxième analyse du tome VI du catalogue de la collection de Michel Bernstein », Annales des Études de la Révolution française et de la Collection des documents de Michel Bernstein, Institut pour le Développement de l'Intelligence Sociale de l'Université Senshu. Le Centre d'Étude des Documents de la Révolution française, 2009 (analyse + catalogue, 208 p.).

(5) Yoshiaki ÔMI, « Cahier de doléances du tiers état de la ville du Puy, élaboré au stade initial du processus électoral (version corrigée et commentée) », Cahiers de la Haute-Loire. Revue d'études locales, 2009, p. 189-203. 
lettre de 1834 dans laquelle il expose le sort qui lui a été réservé à la Restauration).

Yoshiaki ÔMI 1-20-11, Ichikawa, Ichikawa-shi Chiba, 272-0034 Japon jacques3@sj9.so-net.ne.jp

Michel BIARD 61 rue Lord Kitchener 76600 Le Havre biard.m@wanadoo.fr 


\title{
Lettre adressée par Michel Bernstein à l’Université Senshu
}

\author{
« Mémoire sur ma collection révolutionnaire. \\ Par Michel Bernstein ${ }^{6}$
}

\section{Le 7 mars 1979}

Je suis né à Lyon en 1906, de parents russes. Mon père ${ }^{7}$ qui, encore très jeune, avait participé au mouvement révolutionnaire, avait été le plus jeune des délégués au second congrès du Bund (Union des ouvriers socialistes de Russie) $)^{8}$ en 1897 , avait dû, après des condamnations, quitter la Russie des tzars, et s'était réfugié, d'abord en Suisse d'où il fut expulsé pour ses activités anti-tzaristes, puis en France. Après avoir exercé diverses professions, dont celle d'ouvrier typographe - tout en publiant des articles dans les revues libérales russes et dans des périodiques révolutionnaires à l'étranger -, il devint rédacteur pour les affaires russes d'un des plus grands et des plus estimés quotidiens de France : Le Progrès de Lyon.

Lors de la Première Révolution russe, il traduisit du français en russe deux ouvrages importants: l'Histoire du mouvement social en France au XIX $x^{e}$ siècle, du professeur Georges Weill' ${ }^{9}$, et Les Orateurs de la Révolution, du grand historien Alphonse Aulard ${ }^{10}$. Les traductions furent

(6) L'original de cette lettre, envoyée par Michel Bernstein à l'Université Senshu, a été dactylographié avec une machine à écrire dont la frappe semblait parfois capricieuse, ce qui rend aujourd'hui le texte parfois difficile à lire. Plutôt que de publier cet original, j'ai fait le choix d'en donner une copie saisie et annotée (toutes les notes de la lettre, comme celles de l'introduction, sont de Michel Biard). Le texte d'origine a bien sûr été strictement respecté. Je remercie ici ceux et celles qui m'ont apporté des lumières sur tel ou tel détail permettant de mieux comprendre ce précieux document, notamment mon collègue et ami Jean-Numa Ducange.

(7) Léon (Lev Borisovitch) Bernstein (1877-1962). Un fonds Léon Bernstein, fort d'environ 5000 titres, concernant les mouvements révolutionnaires russes, se trouve lui aussi aujourd'hui au Japon, conservé à la Bibliothèque de l'Université d'Hokkaido, sous le nom de « Bernstein Collection ». Vendu une première fois aux États-Unis en 1956 (Newberry Library, Chicago), puis de nouveau en 1965 (Hunter College, New York), ce fonds a été ensuite revendu et acheté en 1984 par le Hokkaido University Slavic Research Center. Le fonds rassemblé par le père achevait ainsi son périple dans le pays où le fils avait vu quelques années plus tôt partir sa propre collection.

(8) Le Bund est un mouvement socialiste créé dans l'Empire russe en 1897 pour rassembler les travailleurs juifs (il est également appelé Union générale des travailleurs juifs de Lituanie, de Pologne et de Russie). Léon Bernstein, alors dans sa vingtième année, en est l'un des membres fondateurs. Défavorable au sionisme, le Bund est peu avant la révolution de 1917 lié aux Mencheviks et opposé aux Bolcheviks.

(9) Georges WeILl, Histoire du mouvement social en France, 1852-1902, Paris, F. Alcan, 1904.

(10) Alphonse Aulard, Les orateurs de l'Assemblée constituante. L'éloquence parlementaire pendant la Révolution française, Paris, Hachette, 1882; Les orateurs de la Législative et de la Convention. L'éloquence parlementaire pendant la Révolution française, Paris, Hachette, 1885-1886 (2 vol.). 
saisies par la police impériale, partie dès la mise en vente, partie à l'imprimerie $^{11}$.

Parmi les écrits de mon père de cette époque, je mentionnerai un très important travail sur l'éducation publique pendant la Révolution française ${ }^{12}$. Ceci explique que, dans la petite bibliothèque de mon père, une grande part était réservée aux ouvrages sur la Révolution française. Dès que je fus en âge de lire des livres sérieux, mon père me laissa prendre ceux de Jaurès, d'Aulard, d'Albert Mathiez, qui furent longtemps mes livres de chevet. Je me passionnais pour cette période, tantôt pour les Girondins avec Michelet, tantôt pour la Montagne et Robespierre avec Louis Blanc et Ernest Hamel...

Les événements qui suivirent en Russie la Révolution de février 1917 changèrent la situation de ma famille. Surtout au point de vue "économique". Tous les journaux dont mon père était le correspondant en France furent interdits dès le début de $1918^{13}$. Il ne resta à mon père que quelques collaborations intermittentes dans la presse française, en dehors du Progrès de Lyon à l'ombre de qui nous vivions.

Des amis d'hier étaient, nombreux, devenus des ennemis politiques, mon père ayant nettement pris position en faveur de la poursuite de la guerre et de la victoire des Alliés. Et des adversaires d'hier étaient devenus proches dans la lutte contre le bolchevisme.

$\mathrm{Au}$ lieu de poursuivre mes études, après avoir passé mon baccalauréat, je fus dans l'obligation de gagner mon pain et d'aider ma famille à vivre. Après différents essais et des travaux passagers, j'entrais au service de l'Agence en France de la Flotte marchande soviétique ${ }^{14}$. L'U.R.S.S. venait d'être reconnue de jure et de facto par la France ${ }^{15}$, et parmi les premiers délé-

(11) ВЕЙЛЬ Ж. (=WeILl G.), ИСТОРИЯСОЦИАЛЬНОГОДВИЖЕНИЯВОФРАНЦИИ 1852-1902 / ПЕР. С ФР. Л.

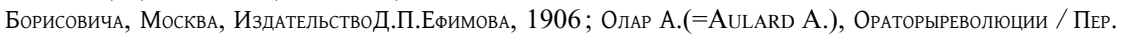
С Фр. Л. БорисовичА, t. 1-2, МосквА, ИзДАтельство Д.П. ЕфимовА, 1907-1908. Dans les deux cas, la traduction est attribuée à Lev Borisovitch, donc sans que le nom de Bernstein apparaisse, afin de duper censeurs et policiers.

(12) Probablement s'agit-il de ce texte : Léon BERnstein, « Un plan socialiste sous la Révolution française », International Review for Social History, 1937, n² 2, p. 193-228 (d'autant que cette revue est publiée par l'Institut pour l'Histoire Sociale d'Amsterdam, auquel il collabore à partir de 1935 [cf. infra, note 23]). Parmi les écrits en question, figurent plusieurs ouvrages sur la Russie, mais aussi un petit livre consacré à la Révolution française publié aux lendemains mêmes du $150^{\circ}$ anniversaire de 1789 : Léon BernsteIn, L’Esprit public en 1789, Paris, sn, 1940.

(13) De toute évidence en raison de l'opposition de ces journaux aux Bolcheviks.

(14) Dans le cadre de la Délégation commerciale soviétique en France, au moment où, profitant des retombées de la NEP, les échanges commerciaux franco-soviétiques se développent (cf. Stanislas JEANNESSON, « La difficile reprise des relations commerciales entre la France et l'URSS [1921-1928] », Histoire, Économie et Société, 2000, n 19-3, p. 411-429).

(15) Cette reconnaissance date du 28 octobre 1924. 
gués envoyés à Paris se trouvaient de nombreux "anciens amis" ou "anciens élèves" de mon père. Très vite, j’y fis mon chemin et parvins à une excellente situation, où je gagnais très largement ma vie nonobstant mon jeune âge.

Pendant ce temps, mon père m'initiait de plus en plus à l'histoire de la Révolution française, époque cruciale dans l'histoire de l'humanité : fin d'un monde, début d'une ère nouvelle. Et je me passionnais de plus en plus pour cette période et les hommes qui la firent.

Dès 1922, le gouvernement bolcheviste avait fondé à Moscou un Institut destiné à étudier l'histoire de la pensée sociale, et principalement du marxisme. Le directeur de cet Institut était le grand historien David Riazanov, que mon père connaissait depuis plus d'un quart de siècle ${ }^{16}$.

En 1927, il proposa à mon père d'être le correspondant de cet Institut pour la France. Les opinions politiques de mon père et son activité comme journaliste importaient peu : il fallait avant tout un correspondant connaissant bien l'histoire révolutionnaire russe et l'histoire de la Révolution française comme celle du mouvement social en France.

Pendant presque tout mon temps libre, j'accompagnais mon père chez les libraires et les marchands d'autographes de Paris. Ainsi j'eus souvent l'occasion, en raison des relations de mon père, d'entrer dans des remises où étaient entassées les bibliothèques acquises et non répertoriées, et d'y fouiller, souvent dans un désordre indescriptible. C'est ainsi qu'en 1926, chez le libraire Bosse, je trouvais quelques caisses de brochures principalement de l'époque de la Révolution française. Charles Bosse venait de publier un catalogue sur ce sujet avec les livres reliés de la bibliothèque qu'il avait achetée ${ }^{17}$, et il hésitait devant le travail à faire pour publier un catalogue de brochures. J'achetais le tout - qui provenait des doubles de

(16) Cet Institut, fondé en 1920 à Moscou, est appelé Institut Marx-Engels. David Riazanov, né en 1870 à Odessa dans une famille juive, de son vrai nom David Borisovitch Goldenbach, évolue en Russie dans les mêmes milieux révolutionnaires que le père de Michel Bernstein. Emprisonné pour ses activités révolutionnaires pendant plus de quatre ans dans les années 1890, il est contraint à l'exil en 1900. Rentré en Russie après la Révolution de février 1917, il rejoint les Bolcheviks. Dans le cadre de l'Institut Marx-Engels, il se consacre à la publication des textes de ces deux auteurs, mais aussi de Hegel, Feuerbach, d'Holbach, Diderot, etc. Sous sa direction, l'Institut acquiert des milliers de livres et documents divers, dont de très nombreuses pièces manuscrites, et amasse également des copies de documents consultés dans les grandes bibliothèques et dépôts d'archives hors d'URSS. Une partie de la collection ainsi constituée concerne la Révolution française, notamment le très important fonds Babeuf.

(17) Le libraire parisien Charles Bosse publie plusieurs catalogues de « livres anciens et modernes » dans les années 1920-1930, et notamment, en 1926, un catalogue intitulé Beaux livres anciens et modernes provenant de la bibliothèque du Château de La Brède (...), composé d'ouvrages ayant appartenu à Montesquieu, Montaigne, Malebranche, etc., dispersés lors de deux ventes à Paris, à l'hôtel Drouot, en mai et novembre 1926. Il est très probable que la référence de Michel Bernstein concerne le catalogue de ces ventes. 
la bibliothèque de La Bédoyère, acquise vers le milieu du XIX siècle par la Bibliothèque nationale ${ }^{18}$ - à tempérament, et m'endettais pour 24 mois.

Les années passèrent. Le plus gros de mon salaire passait dans l'achat de livres. Peu à peu, j'appris mieux qui étaient Hébert, Babeuf, Jacques Roux, les Enragés, et combien rares étaient leurs écrits, souvent détruits après Thermidor ou le procès de Vendôme. J'appris l'importance des feuilles volantes et celle des périodiques. Et jusqu'en 1932, faisant parfois concurrence à mon père, $\mathrm{j}$ ' achetais, en profitant de ce qui était proposé à l'Institut Marx-Engels et refusé par lui.

David Riazanov - le stalinisme pointait - fut brutalement jeté hors de l'Institut qu'il avait fondé, dirigé, animé, et dont il avait fait un des centres d'études les plus importants du monde pour l'histoire sociale ${ }^{19}$. Le même jour, mon père recevait un télégramme de l'Institut mettant fin à ses fonctions de représentant en France, et peu après je perdais ma situation à l'Agence en France de la Flotte marchande soviétique.

Que faire? Le monde entier était plongé dans une crise économique sans précédent. Il était inutile pour moi de chercher du travail après huit années de travail avec les "bolcheviks".

Toutes mes économies étaient en livres. Il me restait une solution, devenir - sans en avoir fait l'apprentissage - libraire. C'est ainsi qu'en septembre 1932 j'ouvris une petite librairie, où très rapidement je me spécialisais en histoire sociale, économique et politique.

Bien à contre-cœur, je dus, dans les années difficiles qui suivirent, me séparer de livres que je continue aujourd'hui encore à regretter. Et cependant j'achetais, j'achetais sans arrêt, à crédit, souvent avec de grosses difficultés financières... Toute la librairie française savait que j'achetais les brochures et périodiques de l'époque de la Révolution française. Peu voulaient s'en occuper, en raison du travail que cela demandait. Cette partie de mon stock devenait de plus en plus importante. Et mes livres de lecture étaient principalement des livres d'histoire, des biographies, des

(18) Noël-François-Henri Huchet, comte de La Bédoyère (1782-1861), membre de la Société des Bibliophiles français, vend sa bibliothèque en 1837, puis en constitue une seconde qui est à son tour dispersée après son décès, en février 1862 (Noël FRANCE, Description historique et bibliographique de la collection de feu $M$. le $C^{t e} H$. de La Bédoyère [...] sur la Révolution française, l'Empire et la Restauration [...], Paris, France, 1862). La BNF a également acquis sa collection de quelque quatre mille estampes historiques.

(19) En février 1931, suite à une simple dénonciation portée contre lui par l'un des accusés du procès du « centre contre-révolutionnaire menchevik », David Riazanov est destitué, emprisonné puis déporté à Saratov. Dans un texte publié dans la revue La Critique sociale, en juillet 1931, Boris Souvarine note : « [...] le travail de son Institut fut suspendu, et presque tous ses collaborateurs furent révoqués ». Au moment des grands procès de Moscou, alors qu'il se trouve toujours assigné à résidence à Saratov, Riazanov est de nouveau arrêté en juillet 1937, puis fusillé en janvier 1938. 
thèses, même des dictionnaires et des bibliographies de l'époque révolutionnaire. Je connaissais pour ainsi dire par cœur Hatin et Tourneux ${ }^{20}$.

Je ne pouvais tout garder. Mes moyens ne me le permettaient pas. Je vendis les doubles. Si de nombreuses brochures sont d'une insigne rareté, telles celles de Babeuf, de Hébert, de Marat, des Enragés et autres, parce que détruites au lendemain du 9 thermidor ou du procès de Vendôme, d'autres sont plus courantes, parce qu'imprimées à très grand nombre. Je remplaçais un exemplaire défectueux par un exemplaire meilleur. Je complétais, j'améliorais. D'autre part, comme souvent il m'arrivait d'acheter un ensemble ou un recueil parce qu'il contenait un ou deux titres que je n'avais pas, je possédais une grande quantité de doubles...

Vint la Seconde guerre mondiale.

Mes livres et ma collection révolutionnaires furent sauvés, grâce à de nombreux amis qui les hébergèrent ${ }^{21}$.

À la Libération, le hasard voulut que je n'eus pas à reprendre immédiatement mes occupations de libraire : de septembre 1944 à avril 1948, je participais à la direction ou à la rédaction - cela est dans la famille! - de divers quotidiens ou hebdomadaires ${ }^{22}$. À nouveau, je gagnais très

(20) Eugène Hatın, Bibliographie historique et critique de la presse périodique française ou Catalogue systématique et raisonné de tous les écrits périodiques de quelque valeur publiés ou ayant circulé en France depuis l'origine du journal jusqu'à nos jours (...), Paris, Firmin-Didot, 1866; Maurice Tourneux, Bibliographie de l'histoire de Paris pendant la Révolution française, Paris, Imprimerie nouvelle - Association ouvrière, 1890-1913, 5 vol.

(21) Michel Bernstein fait preuve ici d'une remarquable discrétion sur les années de guerre. Voici ce que note Michel Cordillot dans sa notice du Maitron (op. cit.) : « Militant de la VI ${ }^{\mathrm{e}}$ section du Parti socialiste en 1938, il fut chargé par André Weil-Curiel de défendre la Gauche révolutionnaire lors d'une assemblée générale de la section qui, en présence de 250 personnes, étudiait les sanctions prises par la direction nationale contre la majorité de la Fédération de Paris. Il rejoignit le PSOP après le congrès de Royan, et le quitta avec André Weil-Curiel pour manifester son désaccord avec le maintien d'une ligne pacifiste après l'Anschluss et Munich. Appelé sous les drapeaux, il fut démobilisé à Royan et regagna Paris en septembre 1940. Alerté par un raid de la Gestapo dans l'appartement occupé par son père, Michel Bernstein, qui avait pris contact dès février 1942 avec Philippe Viannay, décida de se cloîtrer dans l'appartement de son épouse Monique Rollin (il y resta 800 jours sans sortir une seule fois) pour y mettre sur pied l'atelier des faux papiers de Défense de la France. Il fabriqua ainsi près de 12000 faux tampons, d'innombrables papiers d'identité, et même de faux timbres poste qui permirent leur acheminement. Il rédigea également à l'usage des résistants un Manuel du faussaire. Peu avant la Libération, à la demande de Défense de la France, Michel Bernstein et Monique Rollin rejoignirent les maquis de Seine-et-Oise nord. Son action de résistant lui valut d'être décoré de la Légion d'honneur et de la médaille militaire ».

(22) Il participe quelques mois aux activités du journal France Soir, puis à Noir et blanc hebdomadaire. Le célèbre quotidien est fondé en novembre 1944 par des hommes issus de la Résistance, à laquelle Michel Bernstein a donc pris une part active dans le mouvement Défense de la France. Ce dernier a publié à partir de 1941 un journal sous le même nom, feuille clandestine animée par Robert Salmon et Philippe Vianney, tous deux ensuite fondateurs de France Soir. Le premier numéro de l'hebdomadaire paraît, quant à lui, en février 1945, et Michel Bernstein y collabore pendant environ deux ans. 
largement ma vie, et de plus disposais d'une voiture automobile. Pour les besoins de ma profession, je circulais dans toute la France, et en profitais pour chercher chez tous les libraires. Je fis à cette époque de grandes acquisitions.

Les années passèrent. La collection révolutionnaire croissait. Par ailleurs, mon père, après avoir été à Paris le correspondant de l'Institut pour l'Histoire Sociale d'Amsterdam de 1935 à 193923, avait accepté d'être le correspondant à Paris de l'Institut Giangiacomo Feltrinelli de Milan $^{24}$, qui venait d'être créé, et pour lequel il fit d'immenses travaux de recherches dans les archives de Paris et de province sur le mouvement babouviste. J'étais aussi le "libraire ordinaire" de cet Institut. Mais l'histoire de la Révolution française n'entrait pas dans le cadre de ses activités, à l'exception du babouvisme. J'aidais mon père, qui, de son côté, m'aidait. Et, en 1948, je repris mon métier de libraire. Tous mes livres furent transportés à Issy-les-Moulineaux, près de Paris, où ma femme et moi possédions une grande maison.

Lorsque nous quittâmes cette maison en 1959 pour nous installer à nouveau à Paris, je fus littéralement épouvanté du volume que représentaient les brochures et périodiques de la Révolution française. Et c'était une masse qui demandait à être soigneusement classée et collationnée...

Obligé, après un grave accident cardiaque en 1966, de restreindre mes activités physiques, je m'occupais de la mise en ordre de ma collection.

J'avais acheté après la guerre ce qui restait de la bibliothèque du marquis d'Estourmel ${ }^{25}$, député du Cambrésis à l'Assemblée constituante;

(23) L'Institut international d'Histoire sociale est fondé en 1935 à Amsterdam et se charge notamment de collecter des archives dont la conservation est alors menacée dans plusieurs pays européens par le nazisme, les fascismes, mais aussi le stalinisme. Outre les fonds issus des XIX $\mathrm{X}^{\mathrm{e}}$ et $\mathrm{XX}^{\mathrm{e}}$ siècles, avec une forte présence de la mémoire ouvrière, syndicale et révolutionnaire, sont rassemblés des documents du XVIII ${ }^{\mathrm{e}}$ siècle.

(24) La Fondazione (ensuite devenue Istituto) Giangiacomo Feltrinelli, créée en 1949 par Giangiacomo Feltrinelli (1926-1972), est un centre de recherches sur l'histoire, les sciences politiques, économiques et sociales. Elle consacre notamment ses travaux et recherches à l'histoire des révolutions et du mouvement ouvrier, publiant à partir de 1952 une revue nommée Movimento Operaio. Par ailleurs, Feltrinelli est également un éditeur majeur en Italie à partir des années 1950. Cette Fondation a de fait rassemblé un important fonds consacré à Babeuf et à ses compagnons.

(25) Louis-Marie marquis d'Estourmel (1744-1823), élu aux États généraux comme député de la noblesse du Cambrésis, siège à l'Assemblée constituante. Après la séparation de celle-ci en septembre 1791, il rejoint l'armée et devient lieutenant général au début de 1792. Écarté comme d'autres officiers nobles en juillet 1793, il est emprisonné comme suspect en septembre et passe quatorze mois en captivité. Sous 1'Empire, il est membre du Corps législatif et chevalier de la Légion d'honneur. 
de celle de Zangiacomi ${ }^{26}$, député à la Constituante, à la Convention, aux Conseils des Cinq-Cents et des Anciens; de celle de Pardoux Bordas ${ }^{27}$, député de la Haute-Vienne à la Législative et à la Convention; de celle de Bigot de Préameneu ${ }^{28}$, qui avait émigré après le 10 août 1792 ; et surtout de celle de La Rochefoucauld-Bisaccia ${ }^{29}$, qui contenait près de 350 volumes reliés, en plus des brochures non reliées.

La mise en ordre, le classement, la recherche, pour les brochures anonymes de l'auteur lorsque Quérard ${ }^{30}$ ou Barbier ${ }^{31}$, Tourneux pour les écrits concernant Paris, ne le mentionnaient pas, etc., m'occupèrent pendant plus de dix années. Je découvris que je possédais, classées dans les "Sections de Paris", deux brochures rarissimes du marquis de $\mathrm{Sade}^{32}$; je pus établir, en les lisant, que quelques brochures concernant les questions constitutionnelles et financières, restées anonymes dans le Catalogue de la Bibliothèque nationale, étaient d'Achille-Nicolas Isnard, l'auteur du Traité des Richesses publié en $1781^{33}$. J'établissais un fichier, j'annotais

(26) Joseph Zangiacomi (1766-1846) n'a siégé ni à l'Assemblée constituante, ni au Conseil des Anciens. En revanche, il a bien été élu de la Meurthe à la Convention nationale, puis au Conseil des Cinq-Cents. Fait baron sous l'Empire, il poursuit sa carrière dans la haute magistrature jusque sous la Monarchie de Juillet.

(27) Pardoux Bordas (1748-1842), élu administrateur du département de la Haute-Vienne en 1790 , puis successivement élu - dans ce même département - représentant du peuple à l'Assemblée législative, à la Convention nationale, au Conseil des Cinq-Cents puis au Conseil des Anciens. Contraint à l'exil comme régicide en 1816, il rentre en France après la Révolution de 1830.

(28) Félix-Julien-Jean Bigot de Préameneu (1747-1825), élu à Paris pour siéger à 1'Assemblée législative, prend la défense de la famille royale au 10 août 1792 . Toutefois, il n'émigre pas, contrairement à ce qu'écrit Michel Bernstein, mais se retire à Rennes, sa ville natale. Arrêté le 16 prairial an II (4 juin 1794), il est ramené à Paris et incarcéré, avant d'être libéré le 9 fructidor (26 août), conséquence de la chute de Robespierre un mois plus tôt. Après Brumaire an VIII, il effectue une belle carrière au service du régime napoléonien, est l'un des auteurs du Code civil, ministre des Cultes de 1808 à 1814 , et se voit comblé de récompenses diverses (membre de la Légion d'Honneur en 1803, comte d'Empire en 1808, etc.).

(29) Nous n'avons pas encore réussi à déterminer avec certitude quel était celui des ducs successifs portant le nom de La Rochefoucauld-Bisaccia qui possédait cette collection.

(30) Joseph-Marie QuÉRARD, La France littéraire [...] Ouvrages polyonymes et anonymes 1700-1845 [...], Paris, chez l'éditeur, 1846.

(31) Antoine-Alexandre BARBIER, Dictionnaire des ouvrages anonymes et pseudonymes [...], Paris, Imprimerie bibliographique, 1806, 4 vol.

(32) Trois documents existent dans la collection Bernstein (tous trois aujourd'hui également conservés à la Bnf) : Pétition de la section des Piques, aux représentans du peuple français, 1793 ; Discours prononcé à la fête décernée par la section des Piques aux mânes de Marat et de Le Pelletier, par Sade, citoyen de cette section et membre de la société populaire, 1793; Question soumise à la décision des États généraux, ou Réponse au mémoire de Louis-Henri-Joseph de BourbonCondé, duc de Bourbon, prince du sang, des marquis de Janson, de Grimaldi, de Sabran, du comte de Sade, des présidents de Lauris, de Jouques \& de Mazenod, se disant députés de la noblesse de Provence, 1789.

(33) Achille Nicolas Isnard, Traité des richesses, Londres - Lausanne, F. Grasset, 1781. 
le Catalogue de l'Histoire de France pendant la Révolution de Martin \& Walter ${ }^{34}$. Je découvris, chez des relieurs qui travaillaient beaucoup pour mes confrères étrangers, des types de reliure qui me plurent.

$\mathrm{Au}$ fur et à mesure des tris, je faisais relier, isolément ce qui me paraissait le plus précieux, en recueils ce que je n'étais pas assez riche pour faire relier séparément. Au fur et à mesure que cela se faisait, je transportais les volumes à Charentenay ${ }^{35}$, où nous possédions une maison de campagne. Ils occupèrent d'abord une, puis plusieurs étagères, une chambre entière, et enfin deux pièces...

Une crainte me hantait : le fichier que j'avais établi, l'exemplaire interfolié et copieusement annoté du Catalogue Martin \& Walter; les ensembles que j'avais formés sur la question des colonies, sur les questions financières, économiques, sur le babouvisme, sur Hébert, Robespierre, Anacharsis Cloots, Camille Desmoulins, et bien d'autres - tout cet effort, toutes ces années de travail allaient-elles se perdre après moi, dans des ventes qui disperseraient mes trésors aux quatre coins du monde, comme ce fut le cas à la fin du XIX ${ }^{\mathrm{e}}$ siècle pour les collections Pochet-Desroches ${ }^{36}$ et de Nadaillac ${ }^{37}$ ? Je savais que ma collection ne pouvait se comparer à celles de la Bibliothèque nationale ou du British Museum, formées dans le courant du XIX ${ }^{\mathrm{e}}$ siècle. Mais quand même, c'était la collection privée la plus importante de notre époque...

(34) André Martin et Gérard WaLter, Catalogue de l'histoire de la Révolution française (...), Paris, Bibliothèque nationale, 1936-1955, 6 vol. Michel Bernstein achète un exemplaire de ce catalogue et l'annote en reportant toutes les références présentes dans sa propre collection, ainsi que le montre très bien le catalogue publié par l'Université Senshu : Catalogue de l'histoire de la Révolution française par Michel Bernstein, comparé avec le Catalogue de la Bibliothèque nationale, par André Martin et Gérard Walter, Kawasaki, Senshu University, 1979, tomes 1-5, 1980, tomes 6-7, 2001, tomes 8-9. Sur les nombreuses difficultés rencontrées pour établir ce très volumineux catalogue, en raison des lacunes mêmes de celui de Michel Bernstein, voir Yashioki ÔMI, « À propos de l'Inventaire de documents historiques de M. Bernstein », What's pastis prologue, $\mathrm{n}^{\circ}$ 1, 2004 (Bulletin du Centre des Études historiques).

(35) Commune de l'Yonne, située au sud d'Auxerre.

(36) Catalogue de livres provenant de la bibliothèque de M. P. D. [...], Paris, Gauthier A. Chossonnery, 1875. Outre cette vente de mai 1875, une seconde vente Pochet-Desroches a lieu à Paris en janvier 1883 (avec notamment la présence d'une collection complète du Tribun du peuple, de Babeuf, alors adjugée pour 415 francs).

(37) Catalogue d'une collection importante sur la Révolution française, pamphlets, journaux, caricatures, affiches, placards, provenant de M. le C $C^{\text {te }}$. de Nadaillac, Paris, Ant. Chossonnery, 1883; Catalogue d'une collection importante sur la Révolution française, pamphlets, journaux, caricatures, affiches [...] provenant de la bibliothèque de M. le c ${ }^{\text {te }}$ B. de Nadaillac [...] Vente du 19 au 29 janvier 1885 [...], Paris, A. Chossonnery, 1885. 
Mon ami Anton Gerits ${ }^{38}$, que je connaissais depuis de longues années, qui savait ce que représentait mon travail, et la quasi impossibilité de refaire de nos jours une bibliothèque semblable, me vint en aide. Il me proposa de s'occuper de la vente en bloc, de mon vivant, et à des conditions normales, et à celle primordiale que le tout resterait par la suite indivisible, de ma collection.

Et c'est ainsi que cette collection est arrivée à Tokyo, à la Senshu University. Les quelques six mille volumes et cartons - sans parler des chemises où se trouvent des documents autographes ou des "petits papiers" -; les brochures; les quelques centaines de volumes de périodiques; les ouvrages de travail : bibliographies, études, biographies, thèses et autres, serviront rapidement à des travaux d'érudition qui, autrement, n'auraient pu être faits qu'à Paris ou à Londres. Cela est pour moi la plus grande satisfaction que puisse avoir un collectionneur à la fin de sa vie...

Et pour le Japon, pour cette puissance économique et intellectuelle qui grandit d'année en année, c'est une acquisition qui me réjouit, et dont je suis absolument certain qu'elle lui sera grandement profitable.

Michel BERNSTEIN 》

(38) Le Néerlandais Anton Gerits s'occupe lui aussi de livres anciens, depuis 1950, d'abord chez Martinus Nijhoff, puis avec sa propre affaire qui existe encore de nos jours : A. Gerits \& Son, Antiquarian Booksellers (Amsterdam). Entre 1985 et 1996, il a été tour à tour secrétaire général, vice-président puis président de l'International League of Antiquarian Booksellers, ce qui atteste son importance dans les réseaux qui opèrent sur le marché du livre ancien. Une brève recherche sur l'Internet permet aussi de mieux comprendre le choix de Michel Bernstein, au-delà des affinités électives liant les deux hommes, puisque Anton Gerits est ainsi évoqué : « He developed a considerable export trade to Japanese university libraries, and became a major supplier of books from Europe to overseas ». 


\author{
Documents concernant Félix Le Peletier \\ (Senshu University, Collection Michel Bernstein) ${ }^{39}$
}

\title{
Mandat d'amener manuscrit (floréal an IV) ${ }^{40}$
}

\author{
«Mandat d'amener \\ De par la loi
}

Nous Nicolas Joseph Jacques Sutat juge du tribunal du Département de seine et oise, faisant fonctions d'officier comme Directeur du juri arrondissement de Versailles,

Mandons et ordonnons à tous exécuteurs de mandemans de justice d'amener par devant nous, en la chambre du Conseil du tribunal, sise à Versailles, rue de la pompe, $\mathrm{n}^{\circ} 20$, en se conforment à la loi le citoyen Pelletier dit $\mathrm{s}^{\mathrm{t}}$ fargeau demeurant porte de Buc, âgé d'environ trente deux ans, taille de cinq pieds cinq pouces, cheveux noirs, sourcils noirs, pour être entendu sur les inculpations dont ledit $\mathrm{c}^{\mathrm{n}}$ est prévenu.

Requerons tous dépositaires de la force publique de preter main forte en cas de nécessité.

À Versailles, le vingt six floréal l'an quatrième de la République française, une et indivisible.

Signé Sutat.

Notifié la présente copie par nous soussigné officier de police de Versailles, y demeurant rue du chenil $\mathrm{n}^{\circ} 8$, au citoyen Pelletier dit $\mathrm{s}^{\mathrm{t}}$ fargeau en son domicil en parlant à la portierre laquelle ces charge de lui remetre le présent; à Versailles ce vingt neuf floréal de l'an $4^{\text {ème }}$ de la République française.

\section{Geoffroy »}

(39) Le dossier Le Peletier, rangé sous la cote Fol. 67.23, comprend douze pièces. Nous avons choisi de publier ici trois d'entre elles. L'orthographe de ces documents, saisis par mes soins, a été respectée (Michel Biard). Sur la décision d'arrêter Félix Le Peletier, voir la thèse de Laurence Constant, Félix Lepeletier de Saint-Fargeau. Un itinéraire, de la Révolution à la monarchie de Juillet, Paris, Jeunes talents, 1995, p. 63-66. Celle-ci mentionne un premier mandat, signé par Carnot, délivré le 17 floréal an IV. Les deux premiers documents ici publiés illustrent bien sûr le cheminement de ce mandat jusqu'à Versailles. Le 29 floréal aussi bien que le 7 prairial, l'officier de police chargé de l'arrestation ne trouve pas trace de Le Peletier; et Laurence Constant note que le phénomène se reproduit ensuite à l'identique.

(40) Fol. 67.23, pièce 1. 


\title{
Mandat d'arrêt imprimé avec parties manuscrites ${ }^{41}$ (prairial an IV) ${ }^{42}$
}

\author{
« MANDAT D'ARRÊT. \\ DE PAR LA LOI.
}

Nous Nicolas Joseph Jacques Sutat

Juge du Tribunal Civil du Département de Seine et Oise

Directeur du Jury de l'arrondissement de Versailles, faisant

Fonctions d'Officier de Police Judiciaire, en vertu de l'article

70 du Code des Délits et des Peines.

Mandons et ordonnons à tous Exécuteurs de mandemens de Justice de conduire à la Maison d'arrêt de l'arrondissement de Versailles, le citoyen Pelletier dit $S^{t}$ fargeau

Demeurant à la Porte de buc

Prévenu de distribution de journaux et libels incendiaires.

Mandons au Gardien de ladite Maison d'arrêt de le recevoir; le tout en se conformant à la Loi.

Requérons tous dépositaires de la force publique, auxquels le présent Mandat sera notifié, de prêter main-forte pour son exécution en cas de nécessité.

À Versailles, ce cinq prairial

l'an quatre de la République une et indivisible.

Signé Sutat, avec cachet et paraphe.

Ledit Mandat d'arrêt a été notifié par moi soussigné pour aitre remis audit $c^{n}$ Pelletier dit St fargeau que je n'ai point trouvé auquel j'en ai laissé copie à sa portière le sept prairial l'an quatre de la République une et indivisible.

Copie conforme

Geoffroy

officier de Police demeurant

Rue du Chenil $n^{\circ} 8$

à Versailles »

(41) Pour permettre au lecteur de les distinguer, les passages manuscrits portés sur ce document imprimé sont ici indiqués en italiques.

(42) Fol. 67.23, pièce 2. 


\section{Lettre de Félix Lepeletier, non datée $[1834]^{43}$}

"À messieurs les Présidents et Membres de la Commission nommée par Ordonnance royale pour mettre à exécution la résolution des Chambres relative aux condamnations politiques sous la Restauration.

Messieurs,

Dans les 100 jours nommé membre de la Chambre des Représentants par l'Arrondissement de Dieppe, Département de la Seine Inférieure, je fus après la seconde rentrée des Bourbons porté sur les listes de proscription par les Ordonnances du 24 juillet 1815 . Trois jours avant la publication de ces ordonnances je voulus me rendre à ma propriété et demeure du Tilleul, commune de Bacqueville, chef lieu de Canton dont j'étais maire réélu par le Peuple, et de plus Président du dit Canton. Mais prévenu par le Préfet de Rouen $\mathrm{M}^{\mathrm{r}}$ de Girardin que je devais être assassiné par la bande de chouans du Duc de Castries le lendemain 23 juillet dans ma propriété du Tilleul, très isolée, ce fut sur ses instances réitérées que j'attendis à Rouen le résultat certain de ce qui se passerait. Effectivement cette bande prévenue que je devais arriver se porta à ma terre le 23 juillet à cinq heures du matin, et ne m'y trouvant point fouilla ma maison, mes greniers, mes granges, à coups de sabres et de bayonnettes et fit force dégâts. [Ajout en marge : Il paraît constant que l'on me destinait le même sort que le $\mathrm{m}^{\text {al }}$ Brune. Telle fut le bruit du pays, et l'opinion du Préfet $\mathrm{m}^{\mathrm{r}}$ Girardin] Je revins de suite à Paris pour me plaindre de ces hommes au Gouvernement des Bourbons. On étouffe mes plaintes par mon arrestation à la Préfecture de Police où je restai trois jours. Puis, conduit par la Police à la Diligence, je fus envoyé en surveillance à Rouen, puis ensuite à Caen jusqu'à l'époque de la loi de janvier 1816, qui fit sortir du territoire les Français portés sur les listes du 24 juillet 1815, les votants et tant d'autres victimes du despotisme ou de la vengeance des Bourbons restaurés pour la seconde fois.

Pendant ma surveillance de six mois à Rouen et à Caen, il ne me fut permis de venir qu'une seule fois passer deux jours à ma terre. Mais en revanche, on mit dans ma maison des officiers et des soldats anglais, qui y restèrent six semaines, dégradèrent mon mobilier très beau, usèrent de mes provisions, vins, lait, bœure, fruits, fourrages, bois et enfin de tout comme choses entièrement à eux; ce qui m'a causé de grandes pertes.

(43) Fol. 67.23, pièce 4. La lettre porte au dos la mention : «Réclamation condamné politique ». Plusieurs autres documents manuscrits du dossier Le Peletier concernent aussi les démarches que celui-ci effectue sous la Monarchie de Juillet. 
Sorti de France par force en janvier 1816, je me retirai à Bruxelles. J'y restai plus d'une année, mais au bout de ce tems poursuivi encore par la Police des Bourbons jusque là, je fus enlevé en trois heures de tems et remis entre les mains de la Police Prussienne à Aix la Chapelle, pour être conduit avec garnison militaire à mes frais sur ma voiture, à Kœnisberg Prusse Ducale [Ajout en marge : ces faits sont à la connaissance de $\mathrm{m}^{\mathrm{rs}} \mathrm{le}$ $\mathrm{m}^{\text {al }}$ Gérard et le député Teste, l'un à Bruxelles alors et l'autre à Liège où je passai]. Mais je résistai au despotisme prussien; et mené toujours avec ma garnison à mes frais d'Aix la Chapelle à Cologne, de là à Coblentz, le Gouvernement Prussien, sans m'entendre, donna ordre dans cette ville de me mettre de force dans ma voiture et de me conduire à Kœnisberg. Un commissaire de Police et six grenadiers furent chargés de l'exécution. Je m'armai d'une paire de pincettes, et je signifiai que je n'entrerais que mort dans ma voiture. Le commissaire fit instruire le Gouverneur Comte Engersleben de ma résistance. Le Prince Royal de Prusse était alors à Coblentz, il fut décidé que l'on me demanderait où je voulais aller? Je répondis à Francfort sur le Mein. Le commissaire Prussien Graccsoi [?]fut chargé de m'y mener. Il me quitta donc à Francfort où je restai jusqu'en 1819, les proscrits étant alors rappelés en France. Pendant mon séjour à Francfort, l'ambassadeur français Rheinard chercha bien encore à me faire envoyer jusqu'en Bohème, mais ses mauvaises dispositions furent vaines.

Vous comprendrez, Messieurs, quelles playes de telles persécutions font aux intérêts. Mais ce n'est rien encore et je dois ajouter les effets qu'elles produisirent sur ma fortune et mes propriétés dans ma patrie.

J'avais formé dans ma propriété du Tilleul des établissements d'agriculture en grand, un haras de chevaux et une fromagerie à la manière de Gruyères en Suisse. Cette importation couronnée par le succès m'avait valu la grande médaille d'or décernée par la Société d'Agriculture de la Seine. Il me fallut abandonner tout, vendre dans les tems les plus désastreux chevaux, juments, poulains, vaches, en perdre beaucoup par défaut de présence et de soins, vendre mes récoltes sur pied à vil prix, mon mobilier gâté par les Anglais. Les ennemis occupaient partout la France, l'argent ne circulait pas facilement, la défiance était partout, c'était dans les derniers mois 1815. Ces établissements que j'avais formé m'avaient mis dans la nécessité d'emprunter, il y avait quelques années, une somme de 20000 francs hypothéquée sur ma terre; l'échéance de remboursement arriva dans ces circonstances de 1816. J'étais absent; un proscrit ne trouvait pas à emprunter; il se trouva que l'homme à qui je devais était un royaliste, qui se fit un vrai plaisir de me poursuivre à outrance 
en expropriation. Toutes les créances communes à mes frères, neveux et nièces, sur nos biens de famille vinrent fondre sur ma propriété par vingt procès de parution. Le Tribunal de Dieppe que j'avais, comme Commissaire de l'Empereur dans le Département de la Seine Inférieure après le 20 mars, proposé de renouveller en grande partie, mais qui ne le fut pas, me condamna avec délices dans tous mes procès et avec grands frais. Un ordre très coûteux fut établi par l'effet de toutes ces créances [Ajout en marge: nos amis dans le pays envoyés en exil, mes domestiques poursuivis par les tribunaux, condamnés au bannissement. Voyez le dossier Jacques Pizier, la personne qui s'occupait de mes intérêts, menacé d'exil lorsqu'elle voulait les défendre]. Ma terre ne fut vendue que $340000 \mathrm{f}^{\text {ts }}$. Presque tout passa en payements, en frais de défense, d'ordre, de condamnations, de poursuites, ou dans mes dépenses pendant près de quatre ans à l'étranger. Deux ans après la vente de mon bien mon acquéreur le revendit 660000 francs. Le peu qui me resta, après tant de frais et de dépenses payées en 4 ans, je fus obligé, à mon retour, pour avoir de quoi vivre, de le placer en viager. Enfin moi dont la fortune patrimonialle était en juillet 1815 de 25000 francs de rente, j'en suis en ce jour à n'être plus ni éligible ni même électeur [Ajout en marge : Quelle atteinte plus vive et plus douloureuse pouvait subir un ami de la liberté honoré une fois déjà du choix de ses concitoyens!! J'en appelle à vous $\mathrm{mm}$, honorés presque tous aussi du même caractère!!]. Telle est la position, par l'effet de sa condamnation politique sous la Restauration, d'un vrai Français qui servit constamment la cause de la liberté, qui en outre sous l'Empire fut deux fois mis au Temple, déporté deux ans à l'Île de Rhé, exilé deux ans à Genève et six mois dans sa terre; qui enfin eut son frère assassiné par un garde du corps de Louis XVI le 20 janvier 1793, victime aussi, bien plus grande que lui, pour la cause de la liberté.

Je suis, Messieurs, avec la plus haute considération, votre concitoyen et serviteur

Félix LEPELETIER dans sa soixante septième année et ne pouvant presque plus de servir de ses doigts pour écrire » 